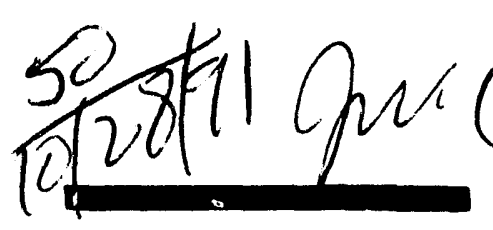

PPPL-2789

UC-420, 427
ARED FOR THE U.S. DEPARTMENT OF ENERGY, UNDER CONTRACT DE-AC02-76-CHO-3073

PPPL-2789

ON RESONANT DESTABILIZATION OF TOROIDAL ALFVÉN EIGENMODES BY CIRCULATING AND TRAPPED ENERGETIC IONS/ALPHA PARTICLES IN TOKAMAKS

BY

H. BIGLARI, F. ZONCA, and L. CHEN

October 1991
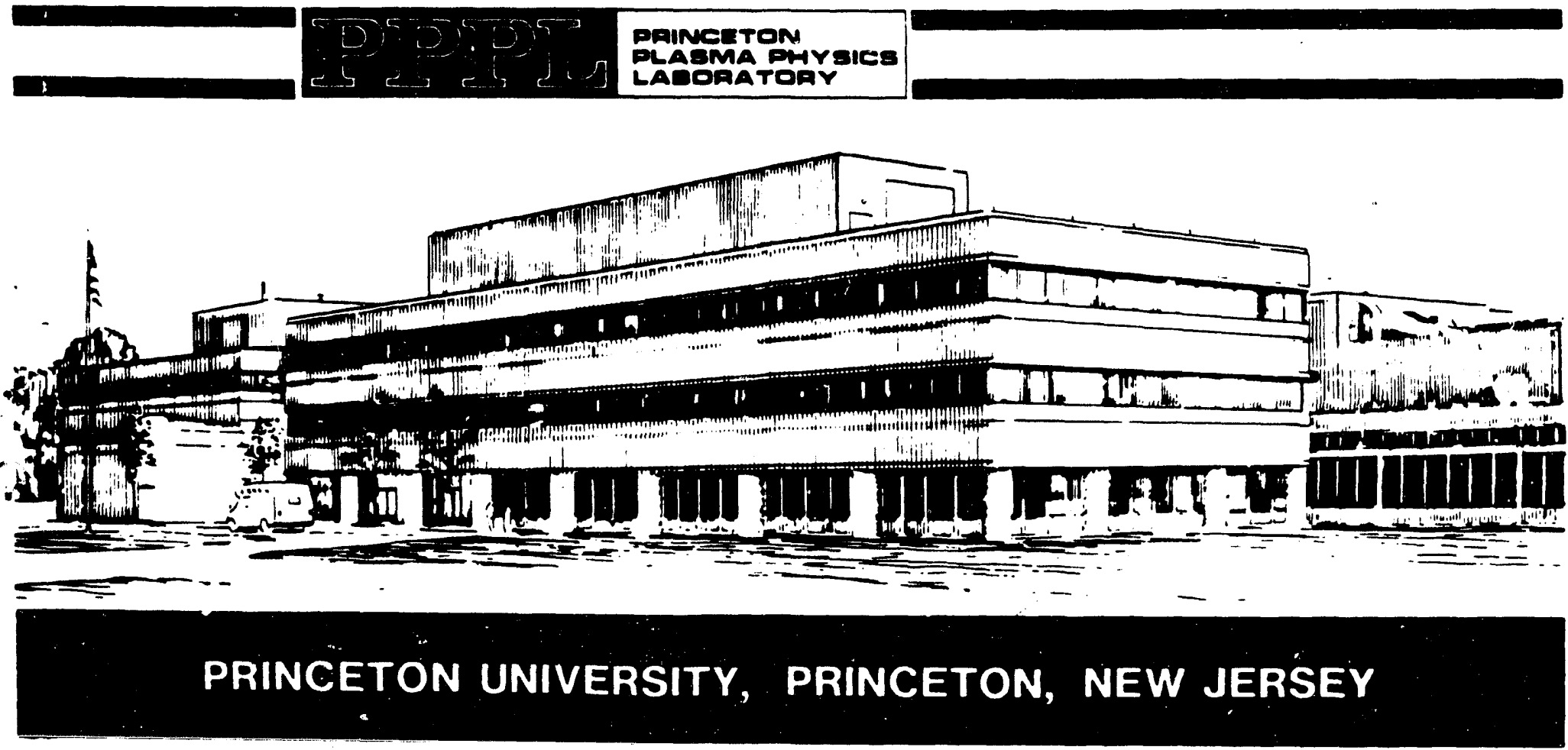


\section{NOTICE}

This report was prepared as an account of work sponsored by an agency of the United States Government. Neither the United States Government nor any agency thereof, nor any of their employees, makes any warranty, express or implied, or assumes any legal liability or responsibility for the accuracy, completeness, or usefulness of any information, apparatus, product, or process disclosed, or represents that its use would not infringe privately owned rights. Reference harein to any specific commercial produce, process, or service by trade name, trademark, manufacturer, or otherwise, does not necessarily constitute or imply its endorsement, recommendation, or favoring by the United States Government or any agency thereof. The views and opinions of authors expressed herein do not necessarily state or reflect those of the United States Government or any agency thereof.

\section{NOTICE}

This report has been reproduced directly from the best available copy.

Available to DOE and DOE contractors from the:

Office of Scientific and Technical Information

P.O. Box 62

Oak Ridge, TN 37831 ;

Prices available from (615) 576-8401.

Available to the public from the:

National Technical Information Service

U.S. Department of Commerce

5285 Port Royal Road

Springfield, Virginia 22161

$703-487-4650$ 


\title{
ON RESONANT DESTABILIZATION OF TOROIDAL ALFVÉN EIGENMODES BY CIRCULATING AND TRAPPED ENERGETIC IONS/ALPHA PARTICLES IN TOKAMAKS
}

\author{
H. Biglari, F. Zonca ${ }^{\dagger}$, and L. Chen \\ Plasma Physics Laboratory, Princeton University, P. O. Box 451, Princeton, NJ 08543 \\ PACS Nos. 52.35.-g, 52.35.Qz, 52.35.Bj, 52.5.Fa \\ Toroidal Alfvén eigenmodes are shown to be resonantly destabilized by both \\ circulating and trapped energetic ions/alpha particles. In particular, the en- \\ ergetic circulating ions are shown to resonate with the mode not only at the \\ Alfvén speed $\left(v_{A}\right)$, but also at one-third of this speed, while resonances exist \\ between trapped energetic ions and the wave when $v=v_{A} / 2 l \epsilon^{1 / 2}(l=$ integer, \\ $\epsilon=r / R$ is the local inverse aspect ratio), although the instability becomes \\ weaker for resonances other than the fundamental. The oft-quoted criterion \\ that instability requires super-Alfvénic ion velocities is thus sufficient but not \\ necessary.
}

\footnotetext{
${ }^{\dagger}$ Permanent address: Associazione EURATOM-ENEA, C. R. E. Frascati, 00044 Frascati, It aly.
} 
A critical concern of next-generation fusion experiments will be the interaction of fusion product alpha particles with the background plasma. The importance of the 3.5 $\mathrm{MeV}$ alpha particles produced by the deuterium-tritium reaction stems from the fact that as they thermalize with the bulk plasma particles, they progressively reduce the need for external heating until a point is reached when even in the absence of such heating sources, the plasma continues to "burn" on its own provisions (so-called ignition phase). The promise of thermonuclear self-heating, however, may be hindered by possible collective instabilities induced by alpha particles, and so it is imperative to assess the pctential for these instabilities both theoretically and experimentally. Indeed, many of these instabilities can be simulated already in non-D-T plasmas by suitably energetic neutral beam injection ${ }^{1,2}$ or RF-produced hot ions. There are two classes of instabilities that are believed to be of serious concern to alpha-particle confinement: kinetic ballooning modes $(\mathrm{KBM})^{3,4}$ and toroidal Alfvén eigenmodes (TAE). ${ }^{5,4,6}$ These modes deserve special scrutiny because they are discrete in character $\left[\omega_{K B M} \simeq \omega_{\text {*ip }}\right.$ and $\omega_{T A E} \simeq v_{A} / 2 q R$, where $\omega_{* i p}=k_{\vartheta} \rho_{i} v_{t i} / L_{p i}, L_{p i}^{-1}=-d \ln p_{i} / d r$ is the bulk ion pressure scale length, $\rho_{i}=v_{t i} / \Omega_{i}$ is the ion Larmor radius, $v_{A}=B /\left(4 \pi m_{i} n_{i}\right)^{1 / 2}$ is the Alfvén velocity, and $q$ is the safety factor]. Hence, unlike modes in the MHD continuum, they experience negligible damping due to phase mixing, and are quite susceptible to instability. In recent work, ${ }^{7}$ we have shown that both circulating and trapped energetic ions can resonantly destabilize KBM's. In this Letter, we show that TAE modes can similarly be excited by energetic ion transit and bounce resonances for a sufficiently steep hot ion pressure gradient. In particular, we show that energetic circulating ions can resonate with the TAE mode not only at the Alfvén speed, but also at one-third of this speed, while resonances exist between energetic trapped ions and the mode when $v=v_{A} / 2 l \epsilon^{1 / 2}, l=1,2, \ldots(\epsilon=r / R \ll 1$ is the local inverse aspect ratio), although the instability becomes weaker for resonances other than the fundamental. Thus, the oft-quoted criterion that instability requires super-Alfvénic ion velocities is sufficient but not necessary.

We now outline the derivation supporting these conclusions, stressing the physical ideas behind the calculation rather than the mathematical details. The plasma is taken to consist of two components: a warm core (' $c$ '), which is treated using an ideal MHD description (thus, there is no parallel electric field perturbation, i.e., $\delta E_{\|}=0$, which in turn couples the electrostatic potential $\delta \phi$, to the parallel vector potential $\delta A_{\|}$), and a tenuous, highly energetic component ( ' $h$ '), which is dealt with using gyrokinetic formalism. We assume the plasma lies in the first stability regime with respect to ballooning modes, and adopt the formal ordering $\beta_{c} \sim \epsilon, \beta_{h} / \beta_{c} \sim \epsilon$ (this assumption is easily relaxed), $n_{h} / n_{c} \sim \epsilon^{3}$, and $T_{c} / T_{h}^{\prime} \sim \epsilon^{2}$. Standard manipulations starting with the continuity and momentum equations then yield the following eigenmode equation:

$$
\begin{aligned}
\mathbf{B} \cdot \nabla \frac{1}{B^{2}} \mathbf{B} \cdot \nabla \nabla^{2} \delta \phi & +\frac{\omega^{2}}{v_{A}^{2}} \nabla^{2} \delta \phi \\
& -\frac{8 \pi p_{c}^{\prime}}{B^{2}} k_{\vartheta} \boldsymbol{\kappa} \times \hat{\mathbf{e}}_{\|} \cdot \nabla \delta \phi+\frac{4 \pi}{B^{2}} \frac{\omega}{c} \boldsymbol{\kappa} \times \mathbf{B} \cdot \nabla\left(\delta p_{\| h}+\delta p_{\perp h}\right)=0,
\end{aligned}
$$

where $\left(\delta p_{\| h}, \delta p_{\perp h}\right)=m_{h} \int d^{3} v\left(v_{\|}^{2}, v_{\perp}^{2} / 2\right) \delta F_{h}$ are the parallel and perpendicular compo- 
nents of the hot particle pressure tensor, and $\kappa=\hat{\mathbf{e}}_{\|} \cdot \nabla \hat{\mathbf{e}}_{\|}$is the magnetic curvature. The first term in Eq. (1) is the shear Alfven field line bending term, the second is associated with the ion polarization drift and incomplete charge cancellation between electrons and ions, the third is the convective core pressure ballooning drive (pressure weighting of unfavorable curvature), while the last term is the hot particle pressure drive. The hot particle distribution function is given by $\delta F_{h}=-\left(e_{h} \hat{\omega}_{* h} / m_{h} \omega\right) \delta \phi+\delta g_{h}$, where $\hat{\omega}_{* h}=\left(\mathbf{k} \times \hat{\mathbf{e}}_{\|} / \Omega_{h}\right) \cdot \nabla \ln F_{0 h}$. The first term is the convective hot ion contribution (which in our ordering is subdominant to the core pressure drive), while the second is the hot particle compressional contribution which drives the instability. The latter satisfies the equation

$$
m_{h}\left[v_{\|} \partial_{l}-i\left(\omega-\omega_{d k}\right)\right] \delta g_{h}=Q_{h} e_{h} i \omega_{d h} \delta \phi,
$$

where $Q_{h}=\left(\hat{\omega}_{z h} / \omega+\partial_{E}\right) F_{0 h}$, and $l$ denotes the coordinate along the field line. The essence of the instability can be read off directly from Eq. (2). The energetic particles lock onto the MHD wave [as characterized by the lhs of Eq. (2)], and surrender energy to it from the free energy stored in their gradients (characterized by $Q_{h}$ ) at a rate given by $e_{h} i \omega_{d h} \delta \phi=\mathbf{v}_{d h} \cdot \delta \mathbf{E}_{\perp}$ (since $\delta E_{\|}=0$ in the ideal MHD approximation and $\beta_{c} \sim \epsilon$, parallel exchange of energy is negligible). The rate of this energy exchange mechanism is spatially varying, being more efficient in some regions of space and less so in others. Since the efficiency depends on the spatial structure of the mode, it is necessary to specify the type of MHD mode under consideration before proceeding further. Our focus in this Letter is on TAE modes, which manifest themselves due to the toroidal modulation in $v_{A}^{2}$ in Eq. (1). Adopting a shifted-circle, magnetic surface equilibrium model, ${ }^{10}$ and transforming to ballooning coordinates, we arrive at the following integro-differential eigenmode equation:

$\left[\frac{d^{2}}{d \vartheta^{2}}+\Omega^{2}(1+2 \epsilon \cos \vartheta)+\frac{\Delta}{f(\vartheta)}-\frac{(\hat{s}-\Delta \cos \vartheta)^{2}}{f^{2}(\vartheta)}\right] \delta \psi-\frac{4 \pi \omega}{c^{2} f^{1 / 2}(\vartheta)} \sum_{j=h, e} e_{j} \int d^{3} v \omega_{d j} \delta g_{j}=0$

where $\delta \psi=\delta \phi / f^{1 / 2}(\vartheta), f(\vartheta)=1+(\hat{s} \vartheta-\Delta \sin \vartheta)^{2}, \Omega=\omega / \omega_{A}, \Delta=\beta^{\prime} q^{2} R$ is the Shafranov shift, $\omega_{d j}=\hat{\omega}_{d j} g(\vartheta)$ is the magnetic drift frequency, $\hat{\omega}_{d j}=k_{\vartheta}\left(v_{\|}^{2}+v_{\perp}^{2} / 2\right) / \Omega_{j} R_{0}$, $g(\vartheta)=\cos \vartheta+(\hat{s} \vartheta-\Delta \sin \vartheta) \sin \vartheta, \hat{s}=r q^{\prime} / q$ is the magnetic shear, and the summation takes account of hot particle compressional destabilization and the stabilizing influence of electron Landau damping [if $v_{A} \gg v_{t i}$ (typical case), it can be shown that ion Landau damping is subdominant by a factor $\sim\left(m_{i} / m_{e}\right)^{1 / 2} \exp \left(-v_{A}^{2} / v_{t i}^{2}\right) \ll 1$; otherwise, if $v_{A} \sim$ $v_{t i}$, it is dominant].

Noting that the destabilizing energetic particle contribution is essentially a perturbation to the background MHD wave, we may identify the structure of the TAE mode irrespective of the hot ions. Since the magnetic shear is very weak in the vicinity of the plasma center where the energetic ion pressure profile is steepest, ${ }^{13}$ we may profitably assume $\hat{s}, \Delta \ll 1$ for the remainder of this calculation. Then, two identifiable spatial scales emerge: $a)$ a periodic scale, $\vartheta_{0} \sim O(1)$, characterizing an 'inner' (ideal) region, and b) a secular ${ }^{8}$ scale $\vartheta_{1} \sim O\left(\hat{s}^{-1}, \Delta^{-1}\right) \gg 1$, characterizing an 'outer' (gap) 
region. In the gap region, Eq. (3) is a Mathieu equation, and can physically be thought of as the Schröedinger equation for valence electrons in the potential well of ions in a periodic crystal lattice. ${ }^{9}$ In the absence of the perturbing potential, the electrons are free to propagate and hence the spectrum is continuous; in its presence, the wave is Bragg scattered from the well, a standing wave forms, corresponding to a discrete energy eigenstate and a gap (forbidden band) in the continuous spectrum. This eigenfrequency and the eigenmode solution, to leading order, are respectively given by, $\omega \simeq \pm \omega_{A} / 2$ and $\delta \psi_{T A E}=\exp \left(-\Lambda\left|\vartheta_{1}\right|\right)\left[\Delta^{\prime} \cos \left(\vartheta_{0} / 2\right)+2 \sin \left(\vartheta_{0} / 2\right)\right]$, where $\Lambda=\left(-\Gamma_{-} \Gamma_{+}\right)^{1 / 2}, \Gamma_{ \pm}=$ $\Omega^{2}(1 \pm \epsilon)-1 / 4 \sim O(\epsilon)$, and $\Delta^{\prime}=4 / \pi \hat{s}$.

We now consider how the energetic particles resonantly destabilize the MHD wave. First focus on circulati.? $q$ particles. Substituting the eigenmode expression for $\delta \psi$ into Eq. (2), and solving for the distribution function, we get ${ }^{4}$

$$
\begin{aligned}
\delta g_{h, u}=\frac{i}{2} \frac{e_{h}}{m_{h}} \frac{Q_{h} \hat{\omega}_{d h}}{\omega \omega_{t}} f_{0}^{-1 / 2}\left(\vartheta_{1}\right) & \sum_{l=1 / 2,3 / 2} \frac{\exp \left(-\Lambda\left|\vartheta_{1}\right|\right)}{\left(\omega / \omega_{t}\right)^{2}-l^{2}} \\
& \times\left[\left(i \frac{\omega}{\omega_{t}} C_{l}+l S_{l}\right) \cos l \vartheta_{0}+\left(i \frac{\omega}{\omega_{t}} S_{l}-C_{l}\right) \sin l \vartheta_{0}\right],
\end{aligned}
$$

where $C_{1 / 2}=\Delta^{\prime}+2 \hat{s} \vartheta_{1}, C_{3 / 2}=\Delta^{\prime}-2 \hat{s} \vartheta_{1}, S_{1 / 2}=-2+\Delta^{\prime} \hat{s} \vartheta_{1}, S_{3 / 2}=2+\Delta^{\prime} \hat{s} \vartheta_{1}, \omega_{t}=v_{\|} / q R$ is the transit frequency, $f_{0}=1+\hat{s}^{2} \vartheta_{1}{ }^{2}$, and the subscript (' $u$ ') 'tr' denotes (un)trapped ions. It is immediately clear from Eq. (4) that two resonances are possible: $v_{\|}= \pm v_{A}$, and $v_{\|}= \pm v_{A} / 3$. Physically, these resonances can be understood as follows. The TAE mode frequency, given by $\omega \simeq v_{A} / 2 q R$, is completely determined by bulk plasma dynamics as discussed above. The destabilizing 'force' associated with the energetic particles, on the other hand, is given by $\mathbf{v}_{d h} \cdot \delta \mathbf{E}_{\perp} \propto \omega_{d h} \delta \psi \propto\left(\cos \vartheta_{0}, \sin \vartheta_{0}\right)\left(\cos \vartheta_{0} / 2, \sin \vartheta_{0} / 2\right)$, which generates oscillations at $3 \vartheta_{0} / 2$ and $\vartheta_{0} / 2$. Parametric pumping occurs when the effective transit frequency of the energetic ions, thus given by $v_{\|} / 2 q R$ and $3 v_{i \mid} / 2 q R$, matches the frequency of the MHD wave; hence, the resonances at the fundamental and one-third Alfvén speed. Equation (4) is now substituted into Eq. (3), and the eigenmode equation is solved by asymptotically matching the ideal region solution to the gap region solution. The resulting dispersion relation, to leading order, is

$$
\Gamma_{+} \simeq i \frac{\pi^{2} q^{2} R^{2}}{c^{2} k_{\vartheta}^{2}} \sum_{j=h, e} \frac{e_{j}^{2}}{m_{j}} \sum_{\sigma= \pm} \int_{u} d^{3} v \frac{\omega_{d j}^{2}}{\omega_{t}} Q_{j} v_{\mathcal{A}}\left[\delta\left(v_{\|}-\sigma v_{A}\right)+\frac{1}{9} \delta\left(v_{\|}-\sigma \frac{v_{A}}{3}\right)\right]
$$

Adopting an isotropic, hot ion slowing-down distribution function, $F_{0 h}^{S D}=\left(B^{2} \beta_{h} / 2^{11 / 2} m_{h} \pi^{2}\right.$ $\left.E_{m}\right) E^{-3 / 2}, E_{c r} \ll E \leq E_{m}\left[E_{m}\right.$ in the maximum (birth) energy and $E_{c r}$ is the energy at which the ions change from slowing down on the electrons to pitch angle scattering off the bulk ions], we obtain the following expression for the growth rate:

$$
\frac{\gamma}{\omega_{r}} \simeq \frac{3 \pi \beta_{h, u} q^{2}}{2^{11 / 2} \epsilon^{1 / 2}} \frac{\omega_{A}^{2}}{\omega_{t m}^{2}}\left(\frac{\omega_{A} \omega_{* p m}}{9 \epsilon \omega_{t m}^{2}}-1\right)-q^{2}\left(\beta_{e} \frac{m_{e}}{m_{i}}\right)^{1 / 2}, \quad v_{m}>v_{A}
$$

where $\omega_{r} \simeq \pm \omega_{A} / 2$, frequencies decorated with a subscript ' $m$ ' denote their value at $E=E_{m}=v_{m}^{2} / 2$, and it is assumed that $\epsilon \geq v_{A}^{2} / 2 v_{m}^{2}$. For $v_{A}>v_{m}>v_{A} / 3$, the growth 
is reduced from the value given in Eq. (6) by $3^{4}$, and if $v_{A} / 3>v_{m}$, there is no instability associated with the energetic circulating ions. Several observations can be made about Eqs. (5) and (6). First, instability ensues when the energetic ion pressure gradient [as given by the first term in Eq. (6)] is sufficiently steep as to overcome the stabilizing influence of velocity-space gradients [i.e., the second term in Eq. (6)], electron Landau damping [i.e., the last term in Eq. (6)], and continuum damping (cf. later). Second, the unstable mode rotates in the same direction as the energetic ions. Third, it is those resonant ions with the highest energy that make the dominant contribution to the instability. This is due to $i$ ) the energy (pressure) weighting of the velocity integrals $\left[\hat{\omega}_{d h}{ }^{2}\right.$ in Eq. (5)], and ii) the fact that velocity-space stabilization increases with decreasing energy $\left(\partial_{E} \ln F_{0 h}^{S D} \propto E^{-1}\right)$. This is a unique characteristic of slowing-down distributions and in distinct contrast, for example, to what one would conclude for a Maxwellian distribution function, suggesting that there are important qualitative differences between the behavior of RF-produced energetic ions on the one hand, and NB energetic ions or alpha particles on the other. The most unstable wavelength corresponds to $k_{\vartheta} \rho_{h} \lesssim(\epsilon / 8 q \hat{s}) v_{A} / v_{m}$, yielding $\left(T_{h}=m_{h} E_{m}\right)$

$$
\frac{\gamma_{\max , u}}{\omega_{A}} \simeq \frac{\pi q \hat{s}}{3 \cdot 2^{17 / 2} \epsilon^{1 / 2}} \frac{n_{h, u}}{n_{c}} \frac{T_{c}}{T_{h} \beta_{c}} \frac{R}{L_{p h}}
$$

Next, we consider the destabilization of the TAE mode by trapped energetic particles. Trapped energetic ions contribute symmetrically about $2 \pi$-periodic regions in ballooning space where $-\vartheta_{t}+2 \pi n \leq \vartheta \leq \vartheta_{t}+2 \pi n, n=0,1,2, \ldots$, where $\vartheta_{t}$ denotes the banana orbit turning point. The trapped particle distribution function may be solved for each $n$ by matching the solution for the two legs of the banana orbit (corresponding to different signs of $\left.v_{\|}\right)$at the turning points. The solution near wave-particle resonance is

$$
\delta g_{h, t r} \simeq-\left(e_{h} / m_{h}\right) \tau_{b h} \cot I_{-\vartheta_{t}}^{+\vartheta_{t}} \overline{\omega_{d h} Q_{h} \delta \phi \cos I_{-\vartheta_{t}}^{\vartheta_{0}}},
$$

where $\tau_{b}=2 \pi / \omega_{b}=\oint_{-\vartheta_{t}}^{+\vartheta_{t}} d l /\left|v_{\|}\right|$is the bounce time, $I_{\vartheta_{a}}^{\vartheta_{b}}=\int_{\vartheta_{a}}^{\vartheta_{b}} d l\left[\omega-\omega_{d h}(\vartheta)\right] /\left|v_{\|}\right|$, and an overhead bar denotes a bounce average, $\overline{(\cdots)}=\oint_{-\vartheta_{t}}^{+\vartheta_{t}}\left(d l /\left|v_{\|}\right|\right)(\cdots) / \tau_{b}$. Substituting this expression into Eq. (3), we obtain the dispersion relation, which we write here as a quadratic form [i.e., integrate the eigenmode equation against $\delta \psi_{T A E}(\vartheta)$ ]

$$
\begin{aligned}
\Gamma_{+} \simeq-i q^{2}\left(\beta_{e} \frac{m_{e}}{m_{i}}\right)^{1 / 2}+i \frac{4 \pi^{2} q^{2} R^{2}}{c^{2} k_{\vartheta}^{2}} \frac{e_{h}^{2}}{m_{h}} \sum_{n=-\infty}^{\infty} \sum_{p=-\infty}^{\infty} \\
\qquad \int_{t r} d^{3} v K_{b} Q_{h} \delta\left(\omega_{r}-\bar{\omega}_{d h}-p \omega_{b}\right) \mid \overline{\left.\omega_{d h} \cos I_{-\vartheta_{l}+2 \pi n}^{\vartheta_{0}+2 \pi n} \frac{\delta \psi_{T A E}(\vartheta)}{\left(1+\hat{s}^{2} \vartheta_{1}{ }^{2}\right)^{1 / 2}}\right|^{2}}
\end{aligned}
$$

where $K_{b}=\oint\left(d \vartheta_{0} / 2 \pi\right)(1-\mu B / E)^{-1 / 2}$. Noting that in Eq. (9), $2 \pi n$ is really the secular $\vartheta$ in disguised form, the sum over $n$ should be understood as an integration over $\vartheta_{1}$, while the bounce average is effectively the integration over $\vartheta_{0}$. Two separate issues determine the physics of trapped energetic ion excitation of TAE modes: i) the 'force' associated with the energetic ions which, in turn, determines the spatial locus where wave-trapped particle 
interaction is maximized, and ii) the rescnarsce condition. We consider each of these in turn. First, we note that in the sum over $n, n=0$ yields the contribution from the ideal region, while $n \neq 0$ gives the contribution from the gap region. It may readily be verified that the latter gives a contribution that is of order $\Lambda^{-1} \gg 1$ larger than from that from the former. Next, we note that the precessional drift frequency, to leading order, is composed of two contributions: a periodic contribution, $\omega_{d h, \vartheta} \equiv k_{\vartheta} v_{d h, \vartheta} \propto \cos \vartheta_{0}$, and a secular contribution given by $\omega_{d h, r} \equiv k_{r} v_{d h, r} \propto \vartheta_{1} \sin \vartheta_{0}$. In the gap region of interest, the secular contribution dominates. For deeply-trapped energetic ions, $I_{-\vartheta_{t}}^{\vartheta_{0}} \simeq \pi p\left(1+\vartheta_{0} / \vartheta_{t}\right) / 2$. It is then clear from symmetry considerations that even bounce harmonics will pick out the $\sin \vartheta_{0} / 2$ contribution to $\delta \psi$, while odd bounce harmonics will pick out the $\Delta^{\prime} \cos \vartheta_{0} / 2$ term. Since for weak shear, $\Delta^{\prime} \gg 1$, the latter will dominate. Next, consider the resonance condition. For the $p=0$ case, which corresponds to the precessional magnetic drift resonance $\left(\omega_{r}=\bar{\omega}_{d h}\right)$, the resonant energy is given by $E_{r e s} / E_{m}=\left(1 / 2 q k_{\vartheta} \rho_{h}\right)\left(T_{c} / T_{h} \beta_{c}\right)^{1 / 2}$. In order to tap the most energetic ions, $k_{\vartheta} \rho_{h} Z 1$, and the strength of the instability is sapped. For the bounce resonance $(p \neq 0)$, on the other hand, the instability readily taps the most energetic ions: $E_{\text {res }} / E_{m}=T_{c} /\left(4 p^{2} T_{h} \beta_{c} \epsilon\right)$. The dominant instability corresponds to $p= \pm 1$, and requires $v_{m} \geq v_{A} / 2 \epsilon^{1 / 2}$. This is because $a$ ) higher bounce harmonics bring lower-energy particles into resonance, for which the instability is mitigated as discussed earlier, b) higher bounce harmonics cause more rapid phase-mixing of the bounce-average integrand, and $c$ ) for weak shear (characteristic of the central regions in the plasma where the alpha particle pressure profile is steepest), odd $p$ picks out the dominant contribution. The growth rate can then be straightforwardly calculated:

$$
\frac{\gamma}{\omega_{A}} \simeq 3 \vartheta_{t}^{4} q^{2} \beta_{h, t r} \frac{\omega_{A}^{2}}{\omega_{b m}^{2}}\left(\frac{\omega_{A} \omega_{* p m}}{6 \omega_{b m}{ }^{2}}-1\right)-q^{2}\left(\beta_{e} \frac{m_{e}}{m_{i}}\right)^{1 / 2} .
$$

The maximum growth rate corresponds to $k_{\vartheta} \rho_{h} \simeq\left(\epsilon^{2} / 2 q \hat{s}\right)\left(T_{c} / T_{h} \beta_{c}\right)^{1 / 2}$, and is roughly of the same order of magnitude as for the transit resonance. It is useful to compare these modes with energetic ion destabilized KBM's. ${ }^{7}$ First and foremost, the latter are triggered only locally close to the first stability boundary for ideal ballooning modes, whereas no such restriction pertains to the TAE modes. Second, for isotropic, energetic ions (such as $\alpha$-particles), the two classes of modes have roughly comparable growth rates: $\gamma_{\max }^{T A E} / \gamma_{\max }^{K B M} \sim \epsilon \beta_{c}\left(\beta_{h} L_{p h} / R\right)^{1 / 2}\left(n_{c} T_{h} / n_{h} T_{c}\right) \sim 1$, while for strongly circulating energetic ions (e.g., parallel NB injection), the TAE mode is dominant: $\gamma_{\max }^{T A E} / \gamma_{\max }^{K B M} \sim$ $\left(1 / q \epsilon^{1 / 2}\right)\left(T_{c} / T_{h} \beta_{c}^{3 / 2}\right)\left(L_{p i} / R\right)^{1 / 2}>1$. . Third, electron Landau darnping is dominant over ion Landau damping for TAE modes, while the reverse is true for KBM's. ${ }^{7}$ For TAE modes, however, recent work $^{11}$ has shown that the dominant stabilizing contribution comes from continuum damping, i.e., since the mode is fundamentally two-dimensional in character, translational invariance is broken because of the radial variation of $\omega_{A} \propto$ $1 / q(r) n_{i}^{1 / 2}(r)$. As a result, the gap mode can hit the continuum, resulting in strong Alfvén absorption at the point of intersection. The critical $\beta_{h}$ for instability is then determined from a balance between the growth rates calculated here, and the magnitude of the continuum damping. On the basis of the calculations presented here, we can conclude that the critical $\beta_{h}$ for instability will exhibit an absolute minimum at the 
fundamental resonance, i.e., $v_{m}=v_{A}$ for the transit resonance, and $v_{m}=v_{A} / 2 \epsilon^{1 / 2}$ for the bounce resonance. For birth speeds below this value, $\beta_{h, c r}$ increases because now only the one-third speed resonance for transit ions or higher bounce harmonics for trapped ions can satisfy the resonance condition (there will be local minima at these resonances, but they may be smeared out because of finite mode widths). For birth speeds above this value, the threshold also goes up because now, a) progressively less energetic ions can be tapped by the resonance, and b) compressional stabilization ${ }^{12}$ of the instability comes into play. Finally, it should be mentioned that while in this Letter, we have concentrated on high- $n$ fluctuations, TAE modes also exist at low $n$ values, ${ }^{5}$ and one may ask which class of modes are likely to be the more unstable. Three competing influences determine the most unstable mode number: i) the growth rate increases with $n$; ii) finite Larmor radius and banana width detuning come into play with increasing $n$; and iii) the continuum ('lattice') structure becomes more dense as a function of the plasma radius with increasing $n$, so that continuum damping may become more accessible. In general, we expect moderate $n$ values (e.g., $2 \leq n \leq 5$ ) to be dominant. Since the calculations presented in this Letter are for localized fluctuations, more nonlocal analysis would be required to assess the lower limit of this range.

We now consider the implications of the theory for experiments. The most unfavorable experimental conditions from the point of view of this instability are to operate at high plasma current, low toroidal field, high electron temperature, moderate density (too high a density reduces $\beta_{h}$ by decreasing the slowing-down time, while too low a density will increase $v_{A}$, making both the resonance condition and exceeding the threshold $\beta_{h}$ more difficult to satisfy), high $\beta_{h}$, peaked $\beta_{h}(r)$, and broad $n_{i}(r)$ (i.e., a flat $\omega_{A}(r)$ profile allows the gap structure to extend as far as possible from the plasma center to the wall, thus minimizing continuum damping). In the worst case scenario where the mode does extend to the plasma edge, it may be possible to stabilize low- $n$ modes by external windings. If high- $n$ modes are excited or if the mode exists deep inside the plasma, then feedback stabilization is not an option. 'The results of this Letter show that although the most unstable situation corresponds to super-Alfvénic hot ion velocities, instability is still possible for sub-Alfvénic velocities, albeit in weaker form. Once the resonance condition is satisfied, the instability will be triggered if the region of steep hot ion pressure profile and high $\beta_{h}$ coincides with the location of the TAE mode, which peaks half-way between adjacent mode rational surfaces. At low edge- $q$, for example, low- $n$ modes are not likely to be excited at all if the hot ion pressure profile is strongly localized about the center. Based on simulations of projected D-T experiments to be performed on TFTR, ${ }^{14}$ we expect both $\alpha$ particle-destabilized TAE and KBM's to be excited in the region $0.2 \lessgtr r / a \lessgtr 0.3$, with typical toroidal mode numbers between 2 and 5 .

\section{ACKNOWLEDGMENTS}

This work was supported by the U. S. Department of Energy Contract \#DE-AC0276-CHO3073. 


\section{References}

${ }^{1}$ K.-L. Wong et al., Phys. Rev. Lett. 66, 1874 (1991).

${ }^{2}$ W. W. Heidbrink et al., Nucl. Fusion (in press).

${ }^{3}$ W. M. Tang, J. W. Connor, and R. J. Hastie, Nucl. Fusion 20, 1439 (1980).

${ }^{4}$ L. Chen, in Theory of Fusion Plasmas, Ed. J. Vaclavik, F. Troyon, and E. Sindoni (CRPP, Association EURATOM, 1988), p. 327.

${ }^{5}$ C. Z. Cheng, L. Chen, and M. S. Chance, Ann. Phys. 161, 21 (1985); C. Z. Cheng and M. S. Chance, Phys. Fluids 29, 3695 (1986).

${ }^{6}$ G. Y. Fu and J. W. Van Dam, Phys. Fluids B 1, 1949 (1989); C. Z. Cheng, Phys. Fluids B 2, 1427 (1990); G. Y. Fu and C. Z. Cheng, Phys. Fluids B 2, 985 (1990).

${ }^{7} \mathrm{H}$. Biglari and L. Chen, submitted to Phys. Rev. Letters.

${ }^{8}$ In real space, the secular scale physically corresponds to singular radial variations, i.e., singular $\partial / \partial r$.

${ }^{9}$ D. A. D'Ippolitto and J. P. Goedbloed, Plasma Phys. 22, 1091 (1980).

${ }^{10} \mathrm{~J}$. W. Connor, R. J. Hastie, J. B. Taylor, Phys. Rev. Lett. 40, 396 (1978).

${ }^{11}$ F. Zonca and L. Chen, submitted to Phys. Fluids B; H. L. Berk, et al., submitted to Phys. Fluids $B$.

${ }^{12} \mathrm{~J}$. W. Connor et al., in Proc. 3rd Joint Varenna-Grenoble International Symposium on Heating in Toroidal Plasmas (CEC, Brussels, 1982) Vol. 1, p. 65; M. N. Rosenbluth et al., Phys. Rev. Lett. 51, 1967 (1983).

${ }^{13}$ The radial distribution of $\alpha$ particles is expected to be sharply peaked about the plasma center because of the sensitive dependence of the $\mathrm{D}-\mathrm{T}$ reaction cross section on temperature.

${ }^{14} \mathrm{R}$. Budny et al., submitted to Nuclear Fusion. 
Dr. F. Paoloni, Univ. of Wollongong. AUSTRALIA

Prot. M.H. Brennen, Univ. of Sydnoy, AUSTRALIA

Plasma Research Lab., Australian Nat. Univ., AUSTRALIA

Prot. I.R. Jones, Flinders Univ, AUSTRALIA

Prot. F. Cap, Inst. for Theoretical Physics, AUSTRIA

Prof. M. Heindler, Instiut for Theoretische Physik, AUSTRIA

Prof. M. Goossens, Astronomisch Instituut, BELGIUM

Ecole Royale Militaire, Lab. de Phy. Plasmas, BELGIUM

Commission-European, DG. XII-Fusion Prog., BELGIUM

Prof. R. Bouciquie, Rijksuniversiteit Gent, BELGIUM

Dr. P.H. Sakanaka, Instituto Fitica, BRAZIL

Instituto Do Pesquisas Especiais-INPE. BRAZIL

Documents Office, Abmic Eneroy of Cenada Lud., CANADA

Dr. M.P. Bectynnskj, MPB Tectnologies, Inc., CANADA

Dr. H.M. Skeregerd, Univ. of Sackatchewan, CANADA

Prot. J. Teichmann, Univ. of Montreal, CANADA

Prof. S.R. Sreonivasan, Univ. of Calgay, CANADA

Prof. T.W. Johnston, INRS-Energie, CANADA

Dr. R. Bolton, Contro canadien de fusion megnetique, CANADA

Dr. C.R. Janes., Univ. of Aberta, CANAOA

Dr. P. Lukec, Komenskeho Universzita, CZECHOSLOVAKIA

Tho Lbrerian, Cuthem Laboratory, ENGLAND

Libray, R61, Autherford Appletion Leboratory, ENGLAND

Mrs. S.A. Hutchinson, JET Library, ENGLAND

P. Muthonen. Univ. of Helsinki, FINLAND

C. Mouttel, Lab. de Physique des Nilieux lonisés, FRANCE

J. Radat, CEN/CADARACHE - Bat 506, FRANCE

Ms. C. Rinni, Univ. of loannina, GREECE

Dr. T. Mual, Academy Bibliographic Ser., HONG KONG

Preprint Library. Hungarian Acauemy of Sci., HUNGARY

Dr. B. Das Gupta, Saha Inst. of Nuclear Physics, INDIA

Dr. P. Kaw, Inst. for Plasma Roseerch, INDIA

Dr. P. Rosenau, Israel inst of Tectinology, ISRAEL

Librarian, Intemational Center for Theo Physics, ITALY

Miss C. Do Palo. Associazione EURATOMENEA, ITALY

Dr. G. Grosso, Istituto di Fisica del Plasma, ITALY

Dr. H. Yamab, Toshiba Ros a Devel Center, JAPAN

Prof. I. Kawakami, Atomic Energy Ros. Inst., JAPAN

Prof. K. Nishikenva, Hiroshima Univ., JAPAN
Director, Japan Atomic Energy Research Inst., JAPAN

Prot. S. Itoh, Kyushu Univ., JAPAN

Data and Planning Conter, Nagoya Univ., JAPAN

Prof. S. Tanaka, Kyoto Univ., JAPAN

Library, Kyoto Univ., JAPAN

Prof. N. Inove, Univ. of Tokyo, JAPAN

S. Mori, Technical Advisor, JAERI, JAPAN

O. Mitarai, Kumamoto Inst. of Tectnology, JAPAN

H. Jeong, Korva Advanced Energy Rosearch Inst., KOREA

Prof. D.I. Choi, The Korea Adv. Inst. of Sai. \& Tech., KOREA

Prof. B.S. Liby, Univ. of Waiketo, NEW ZEALAND

Inst. of Plasma Physics, PEOPLE'S REPUBLIC OF CHINA

Librerian, Inst. of Physics, PEOPLE'S REPUBLC OF CHINA Librey. Tainghua Univ., PEOPLE'S REPUBLIC OF CHINA

2. Li, S.W. Inat Physics, PEOPLE'S REPUBUC OF CHINA

Prof. J.A.C. Cebra, Instiuto Superior Tecnico, POATUGAL

Dr. O. Potrus, Al I cuza Univ., Romania

Dr. J. do Villiers, Fusion Sudies, AEC, S. AFAICA

Prot. M.A. Hellberg. Univ. of Natal, S. AFRiCi

C.I.E.M.A.T, Fusion Division Librery, SPAIN

Dr. L SWnfio, Univ. of UMEA. SWEDEN

Lbrery. Royed Inst of Technoboy, SWEDEN

Prof. H. Witholmson, Chalmers Univ, of Tech., SWEDEN

Centre Phys. Des Plasmas, Ecole Polytoch, SWIZERLAND

Bibliotheak, Inst. Voor Plasma-Fysica, THE NETHERLANDS

M. Durgut, Vice Chaimen, Middle East Tech. Univ., TURKEY

Dr. D.D. Ayutov, Siberian Brench of Academy of Sci., USSR

Dr. G.A. Eliseov, Kurchatov Inst, USSR

Librarian, The Ukr.SSR Academy of Scionces, USSR

Dr. L.M. Kovnizhnykh, Inst. of Generd Physics, USSA

Kemforectungsaniege GmbH, Zentrabibliothek, W. GERMANY

Bibliothok, Inst. For Plasmaforectung. W. GERMANY

Prof. K. Schinder, Ruhr-Universitat Bochum, W. GERMANY

Dr. F. Wagner, (ASDEX), Max-Planck-Institut, W. GERMANY

Librarian, Max-Planck-Institut, W. GERMANY

Prof. R.K. Janev, Inst. of Physics, YUGOSLAVIA 

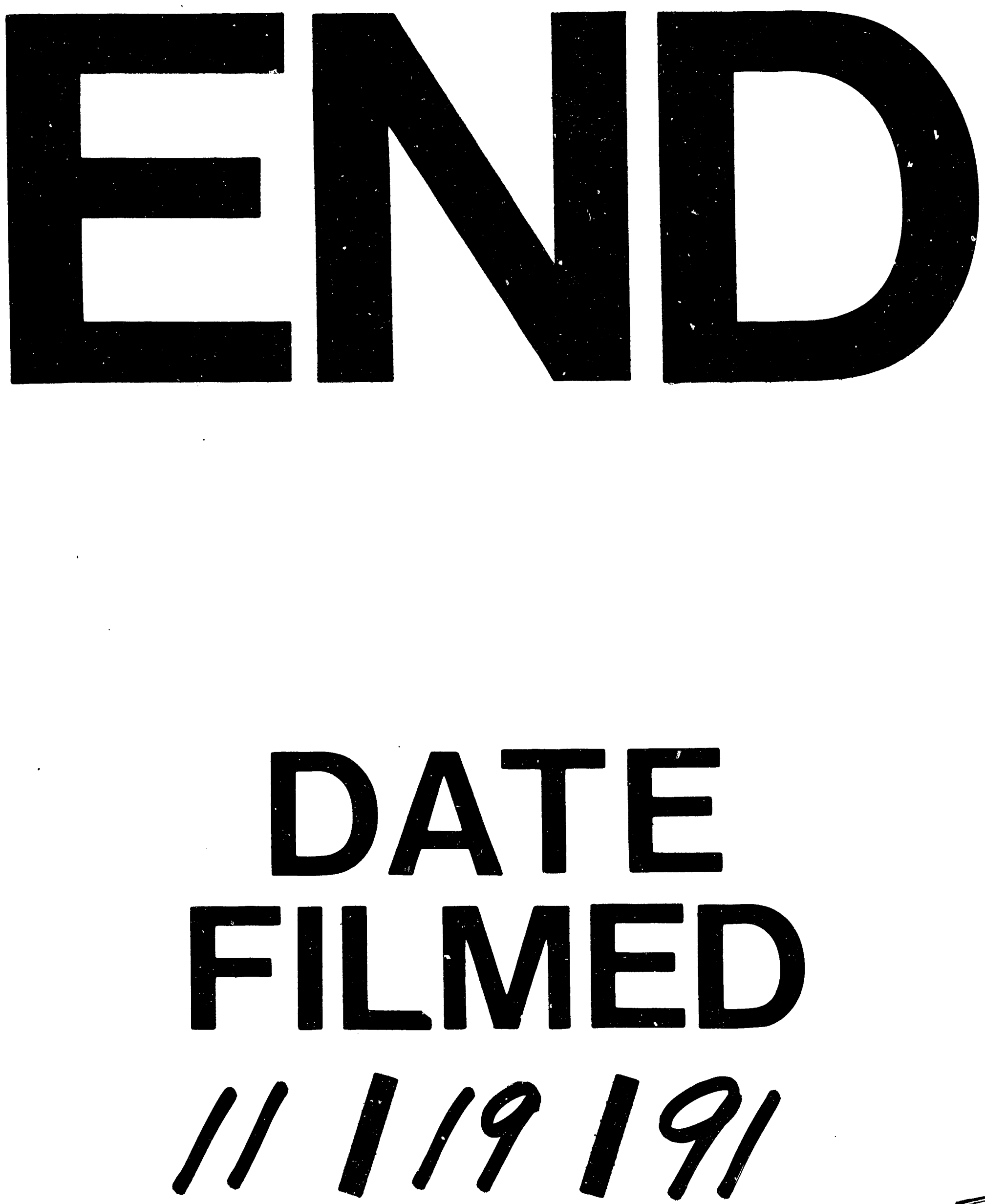

II 